\title{
Observation of ion cyclotron emission from ohmically and NBI heated plasmas in TUMAN-3M tokamak
}

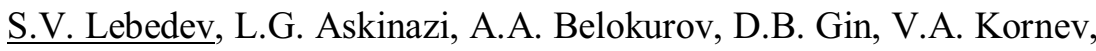 \\ A.A. Shabelsky, A.E. Shevelev, A.S. Tukachinsky, N.A. Zhubr \\ ${ }^{1}$ Ioffe Institute, St. Petersburg, Russia, e-mail: sergei.lebedev@mail.ioffe.ru
}

Ion Cyclotron Emission (ICE) is a fundamental phenomenon observed in space and laboratory magnetized plasmas. ICE exhibit itself as magnetoacoustic instability which develops at frequencies close to ion cyclotron resonance and its harmonics. In laboratory experiments ICE was found to be driven by resonant interaction with high energy ion population produced by fusion products or injected beam ions $[1,2,3]$. Nonmonotonicity or anisotropy of ion distribution function in velocity or physical space are considered as the energy sources for the instability [4].

\section{ICE observation in ohmic plasmas}

In the TUMAN-3M (compact circular tokamak) ICE has been found for the first time in Ohmic regime in the absence of high energy ion population. Frequency of the first harmonic emission was found to be close to IC resonance in the vicinity of pick-up magnetic probe location. Spectra span up to ninth harmonic in deuterium and up to seventh in hydrogen.

Figure 1 presents typical example of ICE spectrum obtained in hydrogen plasma in a shot with the following parameters: $B_{T}=0.8 \mathrm{~T}, I_{p}=150 \mathrm{kA}, n_{e}=1.6 \cdot 10^{19} \mathrm{~m}^{-3}$, $T_{i}(0)=0.2 \mathrm{keV}$. First peak in the spectrum has frequency of $14.7 \mathrm{MHz}$ corresponding to hydrogen cyclotron resonance frequency at probe location. Measurements of the ICE with probe located at low field side of the torus showed reduction of first harmonic frequency in accordance with local ion cyclotron resonance at probe position. Replacement of working gas with deuterium resulted in corresponding reduction of ICE frequencies measured by the both probes.

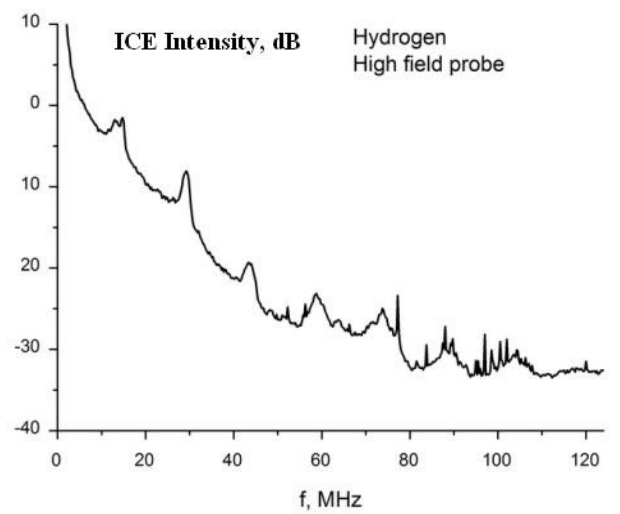

Fig. 1. Spectra of Ion Cyclotron Emission measured in hydrogen plasmas using magnetic probe located at $R=0.42 \mathrm{~m}$ (high field side of the torus)

Ion-cyclotron drift instability (ICDI) [5] is considered as a possible candidate for explanation of the ob- served emission. According to [5] the following criterion should be fulfilled in order to allow ICDI to develop:

$$
\rho_{i} / a \geq 2\left(m_{e} / m_{i}\right)^{1 / 2}
$$

here $\rho_{i}$ - ion Larmor radius, $a$ - radial scale of density/temperature gradient, $m_{e}$ and $m_{i}-$ electron and ion masses, respectively.

Estimations have shown the above criterion can be fulfilled in the experimental conditions of TUMAN-3M tokamak. Strong background turbulence in the spectral range of the observed IC harmonics $(8 \div 120 \mathrm{MHz})$ has been detected indicating existence of energy reservoir for ICDC excitation.

\section{ICE in NBI heated plasmas}

ICE at frequency corresponding to the deuterium cyclotron resonance near magnetic axis of the tokamak was observed during high energy NB injection of hydrogen/deuterium mixture in hydrogen target plasmas. In the described experiments beam energy $E_{0}$ was set to $16 \mathrm{keV}$ and beamline of the injector was aligned in co-current tangential direction with minimal distance between beamline and torus major axis $\mathrm{R}_{\tan }=0.42 \mathrm{~m}$. The emission was detected using pick-up magnetic probes located in both high and low field sides of the torus as seen on figures 2 and 3 . The experiments were performed with the neutral beam containing mixture of deuterium and hydrogen atoms with approximate ratio of $60 / 40 \%$, correspondingly.

It is noticed that replacement of working gas hydrogen with deuterium without changes in beam mixture inverts situation - ICE frequency changes from central deuterium cyclotron resonance to central hydrogen cyclotron resonance. In later case ICE frequency doubles up to $f \approx 12.8 \mathrm{MHz}$.

The phenomenon could be understood in the framework of conjecture "beam-minority-ICE". We assume that beam containing mixture of hydrogen and deuterium ions excites the both hydrogen and deuterium central resonance waves. Depending on target plasma working gas one of the two waves is absorbed inside and cannot be detected by probes located outside plasma. In hydrogen target plasma hydrogen central ICE is absorbed whereas deuterium ICE can escape from the plasma and could be detected. Vice versa: in deuterium target plasma the deuterium ICE is absorbed but hydrogen emission leaves plasma and could be detected outside.

The hypothesis can explain decay of the ICE before NBI termination. As it is seen on figures 2 and 3 the intensity of the "6.4 MHz frequency" decreases towards the end of NBI pulse and disappears at $77^{\text {th }} \mathrm{ms}$ whereas NBI ends at $80^{\text {th }} \mathrm{ms}$. It might be a consequence of accumula- 
tion of deuterium (injected by NBI) in target plasma which results in absorption of "beam-minority-emission".

Other features of the observed IC emission are existence of higher harmonics and fine structure of the first harmonic. Hardware available for measurements of ICE on TUMAN-3M allowed observation of second and third harmonics. These harmonics have larger width and lower intensity compared to first harmonic.

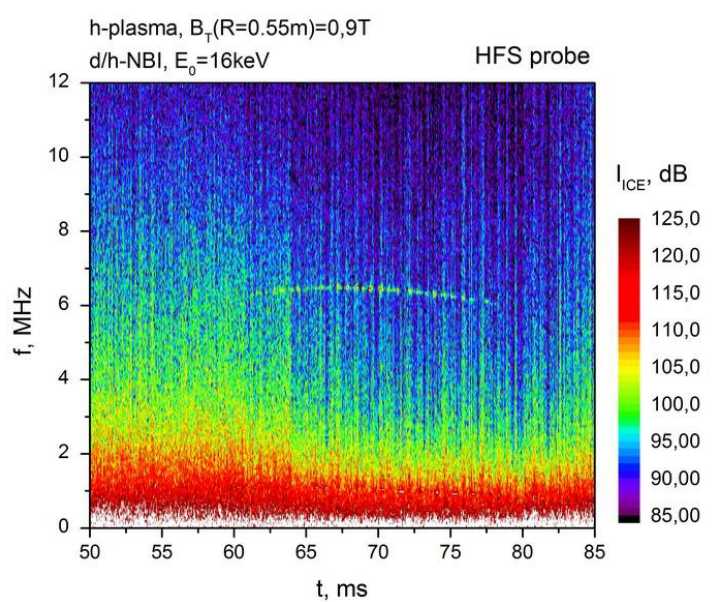

Fig. 2. Spectrogram of plasma emission measured by magnetic probe located at $R=0.42 \mathrm{~m}$ (high field side of the torus). NBI starts at $60^{\text {th }}$ and ends $80^{\text {th }} \mathrm{ms}$. Trace with frequency of $f \approx 6.4 \mathrm{MHz}$ existing during period of injection represents Ion Cyclotron Emission excited by deuterium energetic ions at $R=0.58 \mathrm{~m}$ (close to magnetic axis $R_{0}=0.53 \mathrm{~m}$ )

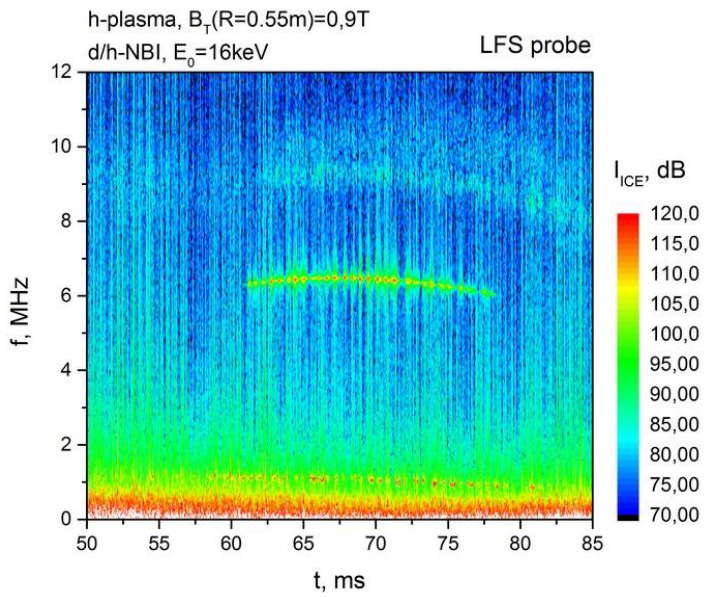

Fig. 3. Spectrogram of plasma emission measured by magnetic probe located at $R=0.77 \mathrm{~m}$ (low field side of the torus) in the same shot as presented on figure 2

It is worth to note splitting of the ICE frequency. Hydrogen minority emission reveals structure of two and in some cases of three thin lines. Observed separation of triplet/doublet lines is in the range of 100-300 kHz. Each component of the fine structure has narrow width of less than $50 \mathrm{kHz}$. Larmor radius of the energetic hydrogen ions is in the range of $0.7 \mathrm{~cm}$ which provides variation of cyclotron frequency of $\sim 300 \mathrm{kHz}$ on this scale. Thus narrow width strongly suggests the observed ICE is not a simple cyclotron emission but rather wave excited in the plasma by energetic ions. A cavity responsible for the splitting and formation of separate narrow lines should exist. For the time of writing this paper the reason of appearance of the fine structure is not clear and needs further studies.

As it was noted above frequency of ICE is close to central cyclotron resonance frequency of either hydrogen or deuterium. In order to establish existence in this area a large amount of energetic ions with high perpendicular energy a calculation of their trajectories in the geometry of TUMAN-3M NBI experiment was performed. In the calculations Monte-Carlo method for generation of ionization points and description of particle density on various trajectories was used. The calculation showed that indeed highest density of energetic ions exists in the proximity of tokamak magnetic axis. For the given amount of test particles the magnitude of $\left(n_{i} V_{\perp}\right)$ in hydrogen is by a factor of 3 higher compared to deuterium. This conclusion correlates with observation of stronger intensity of beam-minority-ICE at hydrogen cyclotron frequency than at deuterium cyclotron frequency.

Results of the calculations can be used for interpretation of splitting. According to the calculations energetic ions arising in the vicinity of magnetic axis produces regions with strong gradient of $\left(n_{i} V_{\perp}\right)$. The regions of the strong gradient are separated by distance of the order of $1 \mathrm{~cm}$ which can explain separation of lines by $300 \mathrm{kHz}$ in fine ICE structure.

In conclusion of this section we summarize new finding made in this study. Conjecture of beam-minority-ICE is proposed for interpretation of transition between emissions at hydrogen and deuterium cyclotron frequencies with change of working gas in the target plasma. Fine structure of ICE was observed for the first time.

Acknowledgement. The study was supported by Russian Science Foundation (Project \# 16-12-10285) and by Ioffe Institute.

\section{References}

1. Bhadra, D. K., Chiu, S. C., Buchenauer, D., Hwang, D. "Electromagnetic emission from a neutral-beam-injected plasma" // Nucl. Fusion. 1986. V. 26. P. 201-209.

2. Cottrell, G. A., Bhatnagar, V. P., Da Costa, O., et al. "Ion cyclotron emission measurements during JET deuteriumtritium experiments" // Nucl. Fusion. 1993. V. 33. P. 1365-1387.

3. Cauffman, S., Majeski R. "Ion cyclotron emission on the Tokamak Fusion Test Reactor" // Rev. Sci. Instrum. 1995. V. 66. P. 817-819.

4. Coppi, B., Cowley, S., Kulsrud, R., Detragiache, P., Pegoraro. $F$. "High-energy components and collective modes in thermonuclear plasmas" // Phys. Fluids. 1986. V. 29. P. 40604072 .

5. Mikhailovsky, A. B. "Ion-cyclotron drift instability in a finite-plasma" // Nucl. Fusion. 1971. V. 11. P. 323-327. 